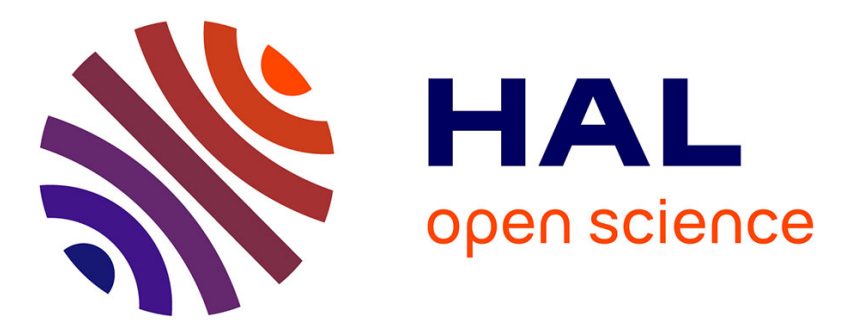

\title{
The role of hot electrons in the dynamics of a laser-driven strong converging shock
}

E Llor Aisa, X. Ribeyre, G. Duchateau, T. Nguyen-Bui, V. Tikhonchuk, A. Colaitis, R. Betti, A. Bose, W. Theobald

\section{- To cite this version:}

E Llor Aisa, X. Ribeyre, G. Duchateau, T. Nguyen-Bui, V. Tikhonchuk, et al.. The role of hot electrons in the dynamics of a laser-driven strong converging shock. Physics of Plasmas, 2017, 24 (11), pp.112711. 10.1063/1.5003814 . hal-01824931

\section{HAL Id: hal-01824931 \\ https://hal.science/hal-01824931}

Submitted on 27 Jun 2018

HAL is a multi-disciplinary open access archive for the deposit and dissemination of scientific research documents, whether they are published or not. The documents may come from teaching and research institutions in France or abroad, or from public or private research centers.
L'archive ouverte pluridisciplinaire HAL, est destinée au dépôt et à la diffusion de documents scientifiques de niveau recherche, publiés ou non, émanant des établissements d'enseignement et de recherche français ou étrangers, des laboratoires publics ou privés. 


\section{The role of hot electrons in the dynamics of a laser-driven strong converging shock}

E. Llor Aisa, X. Ribeyre, G. Duchateau, T. Nguyen-Bui, and V. T. Tikhonchuk Centre Lasers Intenses et Applications, University of Bordeaux-CNRS-CEA, 33405 Talence, France

A. Colaïtis

Centre Lasers Intenses et Applications, University of Bordeaux-CNRS-CEA, 33405 Talence, France Lawrence Livermore National Laboratory, P.O. Box 808, Livermore, California 94550, USA

R. Betti, A. Bose, and W. Theobald

Laboratory for Laser Energetics and Fusion Science Center, University of Rochester, Rochester, New York 14623, USA

(Dated: November 1, 2017)

Experiments on strong shock excitation in spherical plastic targets conducted at the Omega Laser Facility are interpreted with the radiation-hydrodynamics code $C H I C$ to account for parametric instabilities excitation and hot-electron generation. The effects of hot electrons on the shock-pressure amplification and upstream preheat are analyzed. It is demonstrated that both effects contribute to an increase in the shock velocity. Comparison of the measured laser reflectivity and shock flash time with numerical simulations make it possible to reconstitute the time history of the ablation and shock pressures. Consequences of this analysis for the shock-ignition target design are discussed. 


\section{INTRODUCTION}

Shock waves play a major role in inertial confinement fusion (ICF) as a process to control the target implosion and fuel heating [1]. The shocks are excited with temporally shaped laser pulses that ablate the outer surface of the shell and launch a shock propagating inward through the target material. In contrast to a cold piston in classical hydrodynamics, the ablation pressure is defined by the vaporization rate of the shell material, which in turn is defined by the energy transport from the laser-energy deposition zone in an underdense hot plasma to a relatively cold ablation zone. Under the ICF conditions, where the laser intensities exceed $10^{14} \mathrm{~W} / \mathrm{cm}^{2}$, the electrons dominate the energy transport. The energy flux depends strongly on the distribution function of the electrons that are heated and accelerated in the laser absorption zone. It is well known that the classical model of the electron diffusion transport developed for plasmas by Spitzer and Härm [2] and Braginskii [3] is not sufficient: the mean free path of energycarrying electrons could be comparable with the thickness of the transport zone. Nonlocal effects must therefore to be accounted for. The simplest solution consists of introducing a limiter of the diffusion heat flux [4], but more-accurate nonlocal models have also been developed [5].

The nonlocal models provide a good description of the electron energy transport in the cases where the electron heating in the laser field is collisional and the electron distribution function only weakly departs from the local thermal equilibrium. This is not the case, however, for high laser intensities exceeding $10^{15} \mathrm{~W} / \mathrm{cm}^{2}$, where parametric instabilities are excited. Collisionless absorption of the daughter plasma waves results in the production of energetic electrons with characteristic energies exceeding the ambient electron temperature by $10 \times$ to $20 \times$ or more. They can propagate beyond the ablation zone and deposit their energy directly upstream or downstream of the shock. Depending on the ratio of the electron mean free path to the distance to the shock front, the hot electrons may either increase or decrease the shock strength $[6,7]$.

The effect of hot electrons is particularly important for the shock-ignition ICF scheme [8] where a strong shock igniting the fuel is launched at the end of the implosion phase by a strong laser spike with an intensity of the order of $10^{16} \mathrm{~W} / \mathrm{cm}^{2}$. Strong shock experiments have been conducted in the planar and spherical geometries [9], but the shock pressure was not measured directly. It was evaluated with radiation-hydrodynamics codes predicting the time of its propagation across the sample. While such a numerical procedure is constrained by additional measurements of the hot-electron fraction and laser absorption, it is not sufficiently accurate because of a too-simple model of the physics of laser-plasma interaction and hot-electron kinetics.

The record ablation and shock pressures were reported in the experiments preformed at the Omega Laser Facility [10] in the converging geometry $[11,12]$. The $400-$ to $500-\mu$ m-diam solid plastic spheres were irradiated with 60 OMEGA laser beams with a total energy of 22 to $26 \mathrm{~kJ}$, achieving a homogeneous irradiation with an average overlapping intensity of $5 \times 10^{15} \mathrm{~W} / \mathrm{cm}^{2}$ at a wavelength of $351 \mathrm{~nm}$. The 1-ns main pulse was preceded with a 1-ns prepulse of a lower intensity mimicking the plasma corona conditions in the shock-ignition scheme. The laser-driven shock was detected by a short x-ray flash emitted at the moment when the converging shock reached the target center. An earlier flash time corresponds to a stronger shock, higher shock velocity, and higher shock pressure. The shortest flash times were correlated with the generation of a large number of energetic electrons, providing a clear indication of their contribution to shock generation or amplification.

A quantitative evaluation of the shock pressure was performed using numerical simulations with the radiationhydrodynamics code $L I L A C$ [13]. One approach was to adjust the value of the flux limiter [4] to match the x-ray flash time with the observation. This simplified description does not capture important details related to the localization of the energy deposition by hot electrons that can cause target preheat upstream of the shock. Additional simulations were carried out with a fixed flux limiter and a hot-electron transport package [13] to model the interaction [11]. A fraction of the laser energy reaching the quarter-critical $\left(n_{c} / 4\right)$ surface was converted into hot electrons with a single-temperature Maxwellian distribution and $2 \pi$ forward divergence. The electrons were propagated in straight lines into the target with the stopping range calculated by the model described in Ref. [14]. The measured hot-electron fraction and temperature were used as input in the simulations as well as the temporal dependence of the hot-electron production rate. An additional constraint came from the measured absorbed laser power. This model does not selfconsistently describe the laser-plasma interaction in the underdense plasma but rather prescribes a certain energy conversion into hot electrons at $n_{c} / 4$.

A model of laser-energy deposition that takes into account the excitation of parametric instabilities in the plasma corona and hot-electron production has been implemented recently in the radiation-hydrodynamics code $\mathrm{CHIC}$ [15]. In this model, the standard ray-tracing scheme describing the laser propagation in the framework of geometrical optics [16] is replaced with the paraxial complex geometrical optics (PCGO) [17, 18], where realistic laser beams are represented as a combination of Gaussian beamlets. The PCGO model provides access to the laser intensity in any location in plasma and is well suited to account for nonlinear processes in plasma. The model accounts for the resonant absorption (RAB), stimulated Raman scattering (SRS), and two-plasmon decay (TPD) [19]. Each of these processes is a source of energetic electrons, which are characterized by a temperature, density, direction and angular 
divergence. The electrons from the different sources propagate through the plasma, scatter, and deposit their energy according to the classical continuous-slowing-down theory. The model assures the energy conservation at each time step.

First comparisons of the PCGO model with experiments conducted on the PALS Laser Facility in a planar geometry show quite good agreement for both the hot-electron fraction and temperature [19] and the scaling with the laser intensity [20]. It was demonstrated that hot electrons generated by RAB and SRS processes have a relatively low energy, deposit their energy downstream of the shock front, and increase the shock pressure and shock velocity. In contrast, the TPD-generated electrons are more energetic; they penetrate upstream of the shock and preheat the target, which results in a premature expansion. Strong-shock experiments [11, 12] provide a possibility for testing the PCGO model for the first time in the spherical converging geometry and compare it with well-diagnosed data. In what follows, we recall the major experimental results obtained in the strong-shock campaign, describe the principal characteristics of the PCGO model, and present the results of numerical simulations demonstrating the role of hot electrons in the generation and propagation of a strong shock in a convergent geometry. This analysis provides a more-accurate evaluation of the shock pressure under the conditions relevant to the shock-ignition scheme.

\section{EXPERIMENTAL SETUP AND NUMERICAL TOOLS}

\section{A. Experimental results of the strong-shock campaign}

A detailed description of the experiment is presented in Ref. [12]. The main goal was to demonstrate the possibility of generating a shock with a downstream pressure exceeding $300 \mathrm{Mbar}$ in the conditions pertinent to the shock-ignition scheme. OMEGA's 60 laser beams were focused at a wavelength of $351 \mathrm{~nm}$ on a solid spherical target with an average intensity of $5 \times 10^{15} \mathrm{~W} / \mathrm{cm}^{2}$. The laser beams smoothed with phase plates could also be temporally smoothed by spectral dispersion (SSD). In some shots the SSD was turned off, thereby creating a static small-scale intensity pattern at the target surface. This created local zones of high intensity, enhancing the hot-electron production by exciting parametric instabilities. The laser-energy balance was measured with backscatter diagnostics providing the absorbed laser power and time-resolved diagnostics provided temporally streaked spectra of the SRS and the stimulated Brillouin scattering (SBS). SBS is responsible for $\sim 2 \%$ to $3 \%$ of the reflected energy. This fraction did not change significantly between shots with or without SSD. In contrast, the SRS signal was very sensitive to laser beam smoothing. The shots without SSD showed $5 \times$ more SRS scattered light, which persisted during the entire pulse.

(a)

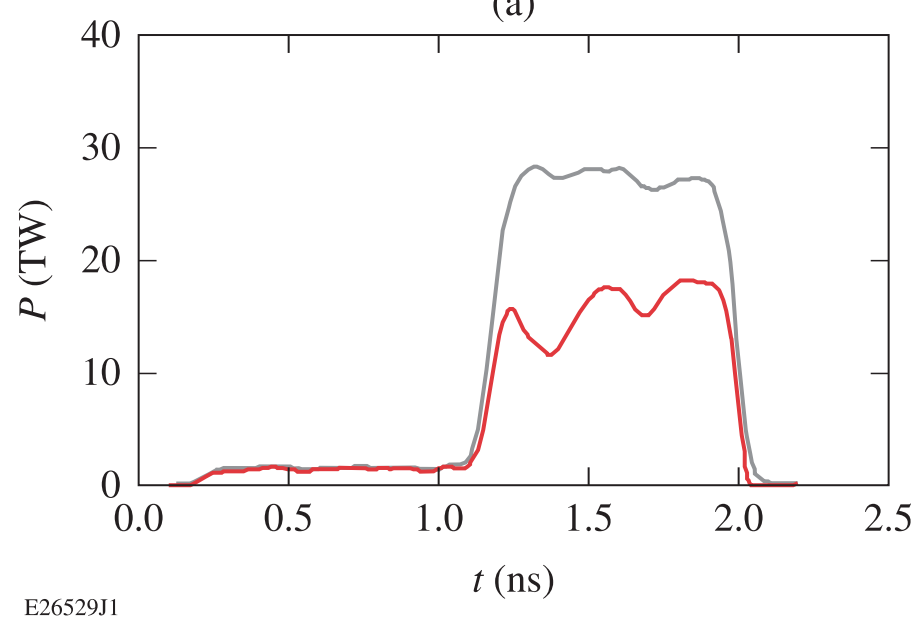

(b)

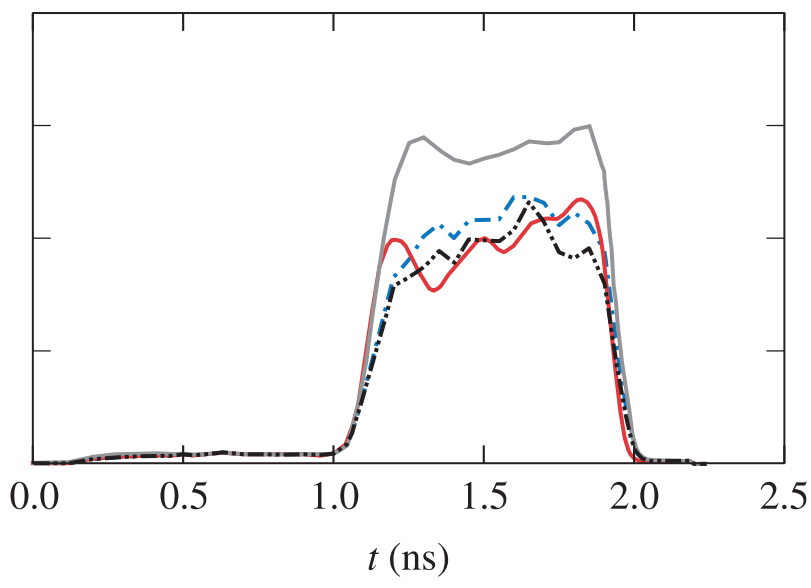

Figure 1. Laser pulse temporal profile at the target (gray) and experimentally measured absorbed power (red) for shots (a) 72676 (SSD on) and (b) 73648 (SSD off). The blue and black curves show the calculated absorbed power with the numerical code $C H I C$ in runs with (blue) and without (black) hot electrons.

Figure 1(a) shows a typical temporal shape of the laser power (gray curve) along with the measured absorbed power (red curve) for the shot with temporally smoothed laser beams. The time integrated absorption fraction, $\alpha$, was measured to be $61 \pm 3 \%$ for this shot (SSD on), which produced a small fraction of hot electrons (3\%). The quantity $\alpha$ is defined as $\alpha=1-E_{351} / E_{L}$, where $E_{351}$ is the measured backscattered energy around the laser wavelength and $E_{L}$ is the measured incident laser energy. Contributions from backscattered SRS were not measured and are not taken 
into account in $\alpha$. Averaging over multiple SSD-on shots, $\alpha$ is slightly higher with $62 \pm 4 \%$. The laser absorption for the similar shot with SSD turned off is shown in Fig. 1(b). This shot produced a much higher hot electron fraction $(\sim 9 \%)$ and exhibited a larger $\alpha(73 \pm 5 \%)$. The average $\alpha$ over multiple SSD-off shots was $71 \pm 3 \%$. The blue and black curves in Fig. 1(b) show the absorption calculated for the shot 73648 with the code $C H I C$ with and without hot electrons, respectively, as explained in the next section. A low-power prepulse operating during the first nanosecond had an intensity below $10^{14} \mathrm{~W} / \mathrm{cm}^{2}$. According to the numerical simulations presented below, it produced a plasma corona with a temperature of $600 \mathrm{eV}$ and a density scale length of $100 \mu \mathrm{m}$ in the near-quarter-critical density zone where nonlinear processes may take place when the 1-ns main pulse interacts with the preformed plasma.

The target had an outer diameter of $480 \mu \mathrm{m}$ for shot 72676 and $496 \mu \mathrm{m}$ for shot 73648 . The target was made out of plastic $(\mathrm{CH})$ that was doped with $5 \%$ of titanium to detect hot electrons and the shock collapse time. The outside was covered with a pure- $\mathrm{CH}$ ablator layer with thicknesses of $34.7 \mu \mathrm{m}$ and $33.9 \mu \mathrm{m}$, respectively. Experiments were also performed with other ablators, but here we consider only the results obtained from $\mathrm{CH}$, which led to the largest number of hot electrons. Other materials were found to be less efficient for hot-electron production [21]. The temperature of hot electrons was in the $60-$ to $70-\mathrm{keV}$ range, increasing by $\sim 10 \%$ when the SSD was turned off. In contrast, the fraction of laser energy transferred to hot electrons increased significantly from 3.3\% when SSD was on to $7.6 \%$ when SSD was off. These values represent averages over multiple shots. The one-to-one correlation between the SRS signal and hot-electron production unambiguously points out to SRS as the origin of hot-electron generation.

The x-ray emission was measured with two instruments - an x-ray framing camera (XRFC) and a streaked x-ray spectrometer (SXS) in the 3- to $7-\mathrm{keV}$ range with a time resolution of $50 \mathrm{ps}$. These instruments detected the emission originating from the target surface and a flash from the target center at the moment of shock collapse. The hot electrons were characterized with three instruments: a four-channel hard x-ray detector (HXRD) having a temporal resolution of $100 \mathrm{ps}$ in the energy range between 20 and $80 \mathrm{keV}$, and two time-integrated diagnostics covering an energy range up to $2 \mathrm{MeV}$. These instruments measured the hot-electron total energy and temperature.

Emission near half of the laser frequency was detected in all shots, indicating excitation of TPD near $n_{c} / 4$. Its signal was much weaker, however, than SRS and did not depend on the laser beam temporal smoothing. TPD is therefore expected to make a minor contribution to the hot electron source, but it generates more-energetic electrons which may contribute to target preheat.

\section{B. Radiation-hydrodynamic code $C H I C$}

The experiment was modeled with the radiation-hydrodynamics code $C H I C$ [15]. CHIC is a 2-D (axial symmetry) arbitrary Lagrangian-Eulerian (ALE) code based on the second-order Godunov numerical scheme. It includes twotemperature (electron and ion) single-fluid hydrodynamics, a Thomas-Fermi ionization model, and $S E S A M E$ tables for the equation of state. The electron and ion energy transport is described by the Spitzer-Härm model with an electron flux limiter and nonlocal electron transport model [5]. The radiation transport is described by a multigroup model with the tabulated opacities calculated in the approximation of local thermodynamic equilibrium. In the original version of $C H I C$, the laser-energy deposition is described by a $3-\mathrm{D}$ ray-tracing model based on the classical geometrical optics [16]. This model accounts for the laser beam refraction in a spatially inhomogeneous plasma and for the collisional absorption by electrons (inverse bremsstrahlung), but it does not fully account for diffraction and is ill suited to compute the laser intensity in plasma [18].

PCGO [22] describes the propagation of a Gaussian beam with two equations: one describes the evolution of the central ray according to the geometrical optics; the other is a complex matrix describing the beam width and the wavefront curvature. In the model implemented in $C H I C$ [17], realistic laser beams are represented by a combination of Gaussian beamlets, each of them described by the PCGO equations. The PCGO model provides access to the laser intensity in any location in a plasma and is well suited to account for nonlinear processes in a plasma. The present 2-D version of PCGO describes the beamlet diffraction in only one transverse direction. The laser collisional absorption is complemented with the resonant absorption (RAB) at the ray's turning point, the TPD instability at the $n_{c} / 4$, and the SRS instability, which was localized near the zone where the plasma density was $20 \%$ of the critical density [19]. The TPD and SRS processes are activated locally as soon as the corresponding threshold conditions are fulfilled. The model predicts the power fraction converted from the incident electromagnetic field to TPD hot electrons. This power is locally subtracted from the optical beamlets and used as input for the hot-electrons beams, ensuring energy conservation. It is assumed that half of the energy is emitted in forward-propagating hot electrons, and the other half in backward-propagating hot electrons. For the SRS process, the power locally subtracted from the optical beamlets is equally divided between backward-propagating light and forward-propagating hot electrons. It is assumed that all three sources produce hot electrons with exponential distribution in energy characterized by a corresponding temperature. The RAB and SRS electrons are generated as collimated beams in the direction of the density gradient in the case of RAB and in the local direction of the pump beamlet in the case of SRS [23], respectively. 
Given the natural spread of wave vectors at the point of emission of SRS (arising from refraction and beam $f$ number), the overall hot-electron emission from SRS is not monodirectional. The TPD electrons are generated in a $90^{\circ}$ wide cone symmetrically with respect to the direction of the pump beamlet. The spatially integrated divergence of the hot electrons (from both TPD and SRS) predicted by the code is in agreement with data from experiments conducted in spherical geometry [24]. The instability conditions and hot-electron sources are updated at each time step assuming that the nonlinear processes are sufficiently rapid to bring the parametric instabilities to saturation on each time step of hydrodynamic evolution $(\sim 1 \mathrm{ps})$.

The hot-electron beam propagation is described in the continuous slowing-down approximation accounting also for pitch angle scattering [25]. The distribution of electrons at the source is split in groups on energy and angle (in the case of TPD) and each group propagates along a straight line. The electrons that reach the plasma edge are allowed to exit, their energy being lost. The electron transport was considered in the stationary approximation, assuming that the electron life time is shorter than the hydrodynamic time step.

\section{C. $C H I C$ simulations without hot electrons}

The strong-shock experiment was modeled with the radiation-hydrodynamics code LILAC [13], which has been used for a long time at LLE to model laser-plasma interaction experiments at lower intensities. As described above, the limitations in $L I L A C$ simulations are overcome here with $C H I C$ by providing a model of hot-electron generation in the plasma corona. To ensure that both codes provide the same description of large-scale plasma hydrodynamics, we performed $C H I C$ simulations for the same laser-plasma interaction physics; i.e., the processes RAB, TPD and SRS were turned off and no hot electrons were generated.

A simulation was performed for shot 73648 (which is equivalent to the shot 72676) but neglected the contribution of hot electrons. The target was a $428-\mu \mathrm{m}$-diam sphere of the $\mathrm{CH}$ doped with $5 \%$ of titanium of $1.72-\mathrm{g} / \mathrm{cm}^{3}$ density covered with a $34-\mu$ m-thick pure-CH layer with a $1.04-\mathrm{g} / \mathrm{cm}^{3}$ density. Since the numerical schemes are different, the same physics may correspond to a different choice of flux limiters. It was found that numerical simulations with both codes gave quite similar results for the shock timing if one used a flux limiter of $8 \%$ in LILAC and $4 \%$ in $C H I C$.

Figure 1(b) shows the temporal profiles of the incident laser pulse (gray) and absorbed power measured (red) and calculated with $C H I C$ (black). The total incident energy was $23.8 \mathrm{~kJ}$. The absorption profile obtained with $C H I C$ is quite close to the measured profile in shot 72676. The calculated absorption of $60 \%$ is in agreement with the experimental value of $61 \pm 3 \%$ for this SSD-on shot but is smaller than the measured average $\alpha$ of $71 \pm 3 \%$ in SSD-off shots. The absorption calculated with $L I L A C$ is $57 \%$, slightly lower than the experimental value for shot 72676 .

(a)

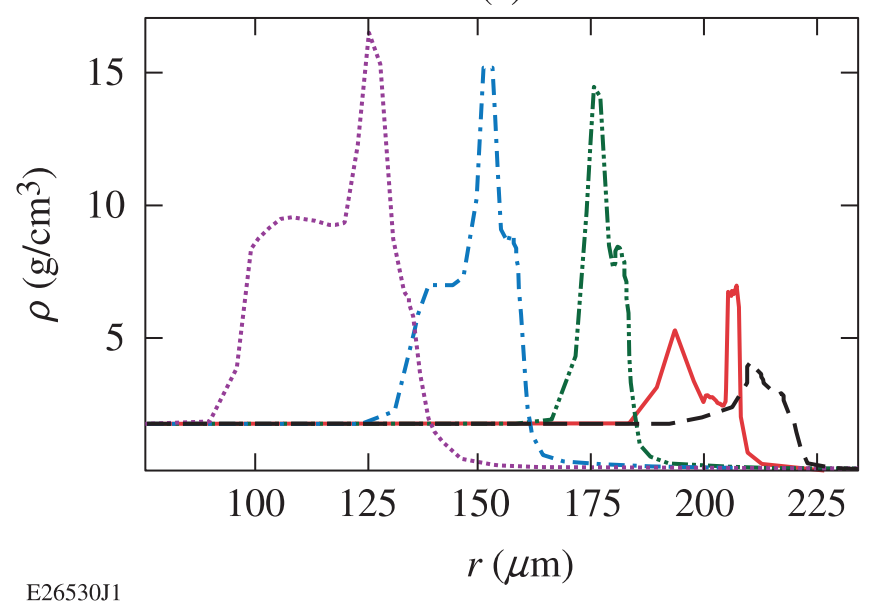

(b)

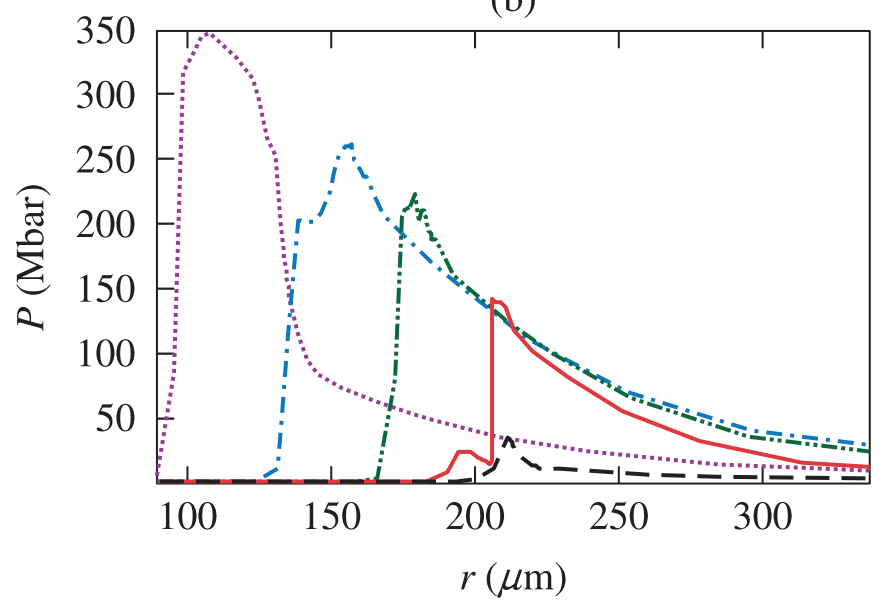

Figure 2. Density (a) and pressure (b) profiles obtained with CHIC simulations for the time interval of the main laser pulse: $t=0.9 \mathrm{~ns}$ (black), $1.2 \mathrm{~ns}$ (red), $1.5 \mathrm{~ns}$ (green), $1.8 \mathrm{~ns}$ (blue) and $2.1 \mathrm{~ns}$ (violet). The time moments correspond to the power profiles shown in Fig. 1.

Figure 2 shows the density and pressure profiles from $C H I C$ in the time interval from 0.9 to 2.1 ns. Similar profiles have been obtained from $L I L A C$. Despite the slight difference in the calculated absorbed energy, both codes give the same shock collapse time of $2.8 \mathrm{~ns}$. At the time when the main pulse arrives, $t=1 \mathrm{~ns}$, the shock created by the prepulse has already traveled through the ablator and entered the denser part of the target. Its pressure is at 
a relatively modest level of 20 to 30 Mbar (black profiles in Fig. 2). The shock created by the main pulse is much stronger. Its pressure rises above $200 \mathrm{Mbar}$ when it catches up with the first shock at $1.5 \mathrm{~ns}$. The pressure enhances to $\sim 300$ Mbar because of the convergent effect at the end of the laser pulse. The shock continues progressing to the center after the end of the laser pulse, and the pressure attains the value of a few Gbar at the moment of collapse.

\section{NUMERICAL SIMULATIONS OF THE CONVERGING SHOCK}

The simulations shown in the previous section demonstrate that CHIC provides similar results to LILAC in the hydrodynamic regime, where the nonlinear laser-plasma interaction processes and hot electrons are not important. We consider these results as representative for the case where the SSD was turned on. In contrast, in the case of SSD off, there are strong fluctuations of laser intensity in plasma, and parametric instabilities can be excited in speckles. Our PCGO model accounts for the SRS and TPD excitation and subsequent generation of the hot electrons. The enhanced laser absorption and hot-electron production facilitate the generation of a stronger shock. It propagates faster and arrives $390 \mathrm{ps}$ earlier at the target center according to simulations. A correlation of the enhanced SRS activity with the hard $\mathrm{x}$-ray diagnostics shows $(9 \pm 1 \%)$ of the incident laser energy converted into hot electrons, indicating the role of hot electrons in shock acceleration. Simulations presented in this section establish relations between the SRS, hot-electron generation, and shock dynamics.

Numerical simulations with $C H I C$ were conducted in a 1-D spherically-symmetric configuration with an electron flux limiter of $4 \%$ (see Section IIC) and with the PCGO module switched on. The previous parameters of the PCGO model were chosen based on a comparison with experiments in the planar geometry [19]. The hot-electron beamlets propagate in the meridional plane with a fixed transverse width.

\section{A. Energy balance}

Simulation results shown in the previous section provide information on laser-plasma interaction conditions. At the moment the main pulse arrives, the plasma is expanded and the critical density is located $240 \mu \mathrm{m}$ from the target center, while $n_{c} / 4$ is $300 \mu \mathrm{m}$ away. The characteristic density scale length at this latter point is $100 \mu \mathrm{m}$, and the electron temperature, which is $0.6 \mathrm{keV}$ before the main pulse arrives, increases rapidly to $3.8 \mathrm{keV}$. These parameters allow us to estimate the contribution of parametric instabilities in the laser-plasma absorption. The collisional absorption of a laser beam on such a plasma profile is $\sim 50 \%$; i.e., the laser intensity near $n_{c} / 4$ is $\sim 70 \%$ of the incident intensity or $3.5 \times 10^{15} \mathrm{~W} / \mathrm{cm}^{2}$. This value is significantly larger than the thresholds for the SRS and TPD instabilities.

Using the expressions for the SRS and TPD thresholds (see, for example, Ref. [9] and references therein), one finds that the laser's prepulse intensity stays below the thresholds of both instabilities. In that case, laser absorption is caused only by the electron collisions. At the moment the main pulse arrives, the SRS threshold is exceeded by $10 \times$, while the TPD threshold is exceeded by only $5 \times$. This ratio between the two instabilities is explained by the fact that the SRS threshold depends only on the density gradient and is mostly unaffected by the main pulse's arrival. In contrast, the TPD threshold is proportional to the electron temperature, which increases rapidly upon the main pulse's arrival. Therefore, rapid plasma heating favors SRS and suppresses TPD.

The repartition of the absorbed energy is shown in Fig. 3. The contribution of the resonant absorption is only important at the beginning of prepulse when the density profile is steep. However, the energy of RAB-generated electrons is relatively small throughout the laser pulse and its contribution to the energy balance is less than $2 \%$. The SRS dominates the interaction. It starts at the arrival of the main pulse and corresponds to an instantaneous conversion of $6 \%$ of the laser power into electrons with the temperature of $40 \mathrm{keV}$. The TPD contribution in the energy balance is smaller, and only half of the electrons are directed to the dense plasma. The second half is directed outwards and does not contribute to absorption. However, the TPD-generated electrons are more energetic, their temperature is $\sim 70 \mathrm{keV}$, and consequently their mean free path is more than $3 \times$ longer than for the SRS electrons.

The total balance of absorbed energy in the simulations is as follows: RAB 1.6\%, SRS $5.4 \%$, TPD $2 \%$ and collisional absorption $60 \%$ (i.e., $69 \%$ of the incident laser energy). This is compared to the measured $\alpha$ of $73 \pm 5 \%$, as shown in Fig. 1(b). A small fraction of stimulated Brillouin scattering amounting to $3 \%$ in the experiment is not accounted for in our simulation. There is also a slight difference in the hot-electron dynamics. The hot electrons in the experiment [21] were observed with a delay of $\sim 200$ ps with respect to the main laser pulse and their instantaneous conversion fraction is larger than calculated. This demonstrates the limits of the PCGO model, which does not account for the temporal evolution of parametric instabilities (other than related to hydrodynamic effects), assuming that the nonlinear saturation and energy transfer take place on a time scale shorter than one time step in the hydrodynamic simulation (of the order of $1 \mathrm{ps}$ ). This energy balance can be compared with the case where the parametric instabilities were switched off; the total absorption is then calculated to be $61 \%$. Therefore, the parametric instabilities accounted 
(a)

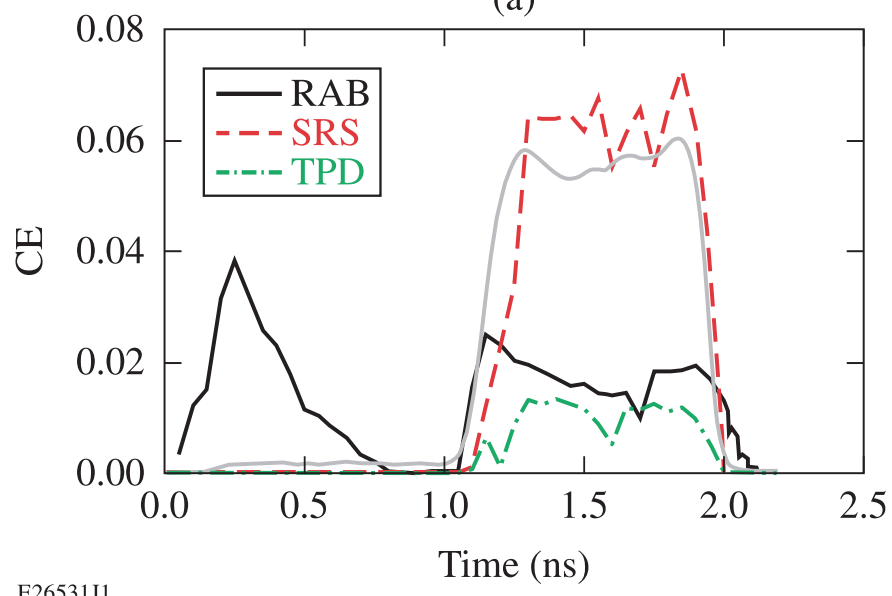

(b)

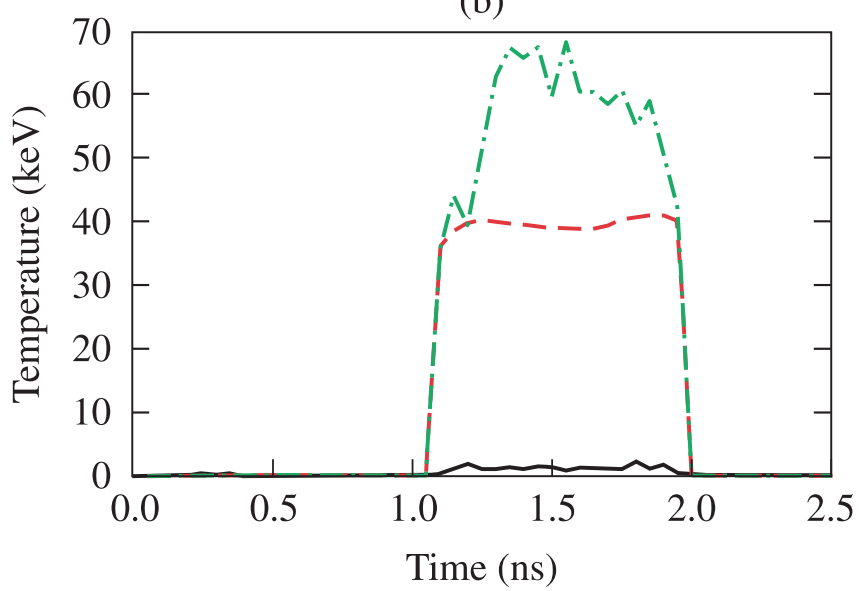

Figure 3. (a) Fraction of laser power converted in hot electrons and (b) their temperature calculated with $C H I C$ for the laser power profile shown with gray line in (a). Resonance absorption is shown in black, SRS in red, and TPD in green.

for slightly reduce the collisional absorption (as smaller laser power attains the near-critical densities) but significantly increase the total absorbed power resulting from the generation of hot electrons.

The reflected energy in the CHIC-PCGO simulation is $31 \%$. It comprises $\sim 11 \%$ SRS and $20 \%$ at the main wavelength. The PCGO model does not account for the SBS, which was $\sim 3 \%$ in the experiment. In the simulations this fraction was subtracted from the incident laser energy. Accounting for the SBS contribution, the calculated reflected energy fraction at the main wavelength is $23 \%$, which is in reasonable agreement with the measured reflection of $27 \pm 5 \%$.

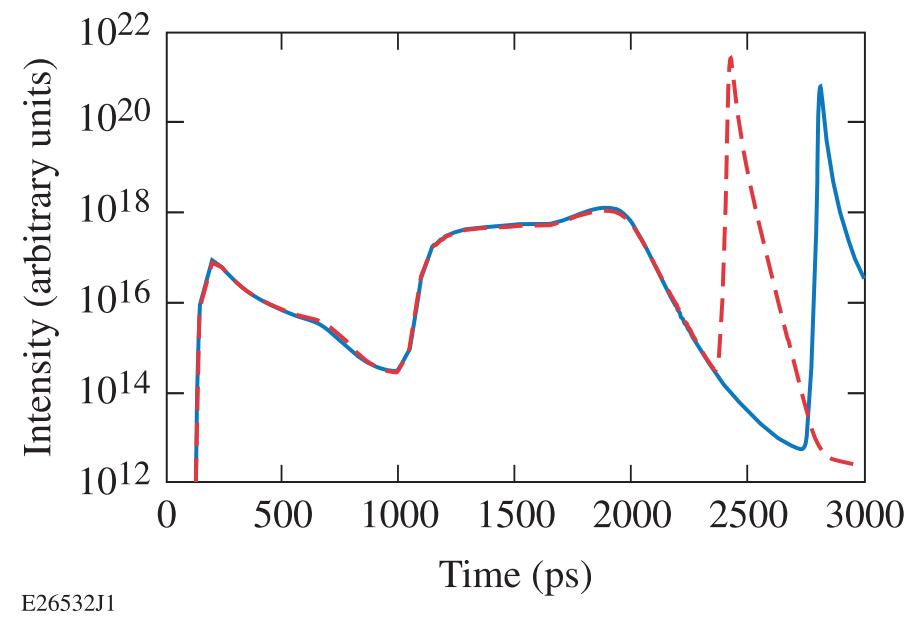

Figure 4. Temporal evolution of the plasma x-ray emission in the 3- to 5-keV energy range obtained from post-processing $C H I C$ simulations with (red) and without (blue) hot electrons.

Although the PCGO model was not specially tuned for this particular experiment, it provides good agreement with the measured shock flash time for the SSD-off case, as discussed below. The x-ray emission of titanium ions in the 3- to 5-keV energy range was calculated with a spectral code TRANSPEC [26] by post-processing the hydrodynamic profiles calculated with $C H I C$. The temporal evolution of the x-ray emission shown in the graph of Fig. 4 has three parts: The first weak emission peak comes at the prepulse arrival, resulting from plasma emission created at the target surface. The second one is correlated with the main pulse. This is also emission of the coronal plasma but with intensity an order of magnitude larger because of higher plasma temperature. There is no measurable contribution of hot electrons at that time. A significant difference can be seen only in the last flash, which is caused by the emission of titanium ions (He- $\alpha$ line at $4.7 \mathrm{keV}$ ). The intensity of the flash is three orders of magnitude higher, and the presence of hot electrons increases the flash intensity in agreement with experiments [12]. The calculated flash time of $2436 \mathrm{ps}$ agrees with the experimentally measured flash time of $2440 \pm 50 \mathrm{ps}$ for the case without SSD. The 
case with SSD was modeled with the PCGO model by simply turning off the parametric instability modules, which is an oversimplification since there is still some degree of hot-electron generation observed experimentally in those cases. Without the hot-electrons modules, the PCGO model does not succeed in reproducing the experimentally measured flash time of $2500 \mathrm{ps}$ for the shot with SSD, predicting a flash time at $2818 \mathrm{ps}$ (blue curve in Fig. 4). This shows that the lower amount of hot electrons observed in that case $(\sim 3 \%)$ is also important and should be accounted for. This case can be considered in the future when the PCGO model will include the SSD option. Nevertheless, the large difference observed in modeling results between the cases with and without hot electrons highlights the importance of consistently accounting for those in radiation-hydrodynamics codes.

\section{B. Shock-wave dynamics}

Numerical simulations also provide information on the contribution of hot electrons to shock-wave dynamics. Figure 5 shows the spatial and temporal evolution of the pressure in the target obtained with and without hot electrons. The first shock with the amplitude of $30 \mathrm{Mbar}$ crosses the ablator and enters the inner target at $t=0.8$ ns. The main pulse arriving at $t=1 \mathrm{~ns}$ generates a stronger shock with a pressure of 130 to $150 \mathrm{Mbar}$, while the ablation pressure is maintained at a level of $110 \mathrm{Mbar}$. In the case without hot electrons, this shock enters the main target and merges with the first shock at $1.5 \mathrm{~ns}$. During merging, the shock pressure slightly decreases and then increases again because of the convergence effect. The shock collapses in the center at $2.8 \mathrm{~ns}$.

(a)

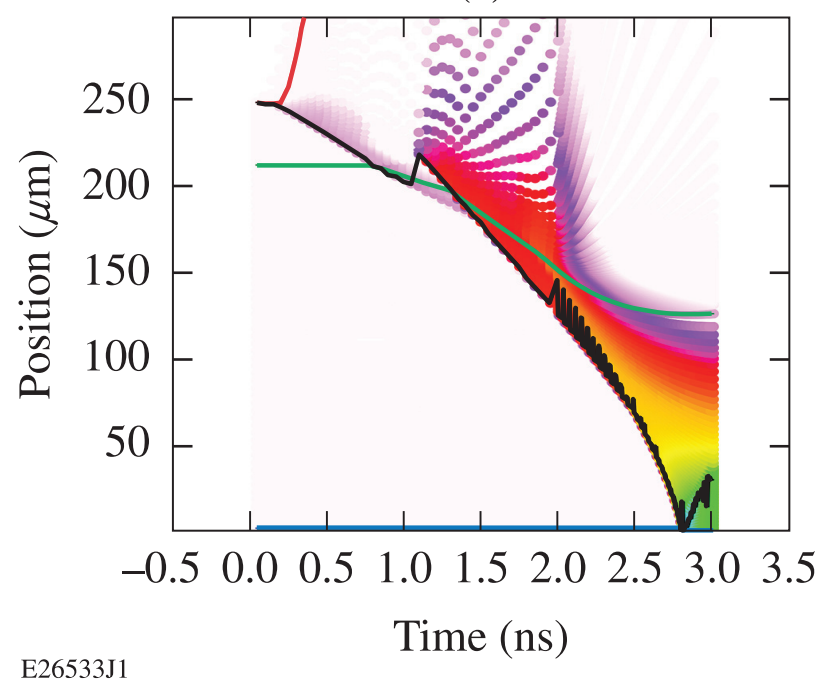

(b)

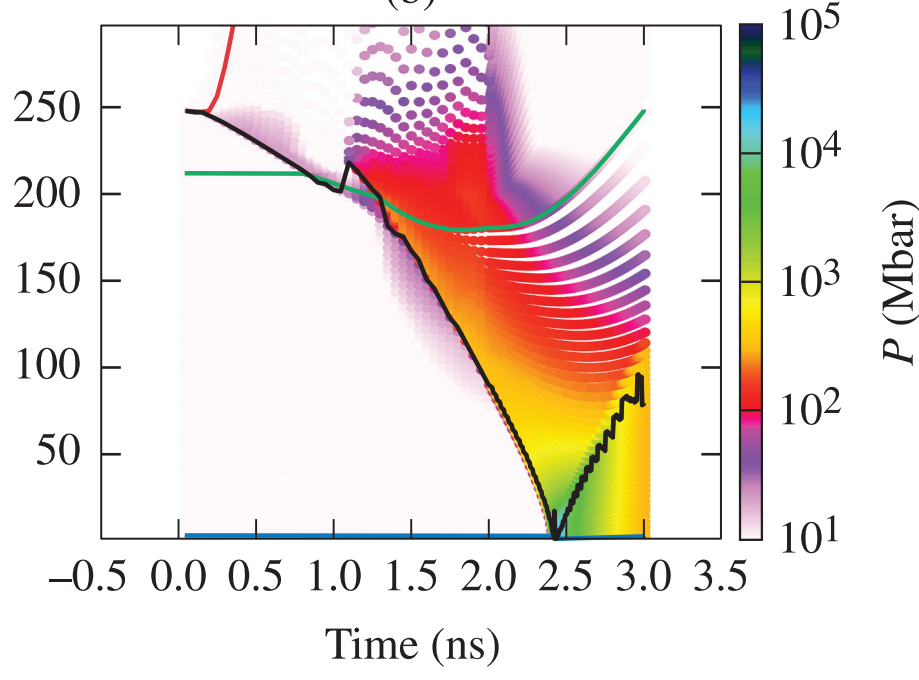

Figure 5. Pressure evolution as a function of the position in the target and time for the case (a) without hot electrons and (b) with hot electrons. The red line shows the position of the external target surface, the green line the interface between the ablator and the inner part, the black line the position of the strongest shock.

The temporal evolution of the pressure at the shock front and the ablation pressure is shown in Fig. 6 . The position of the ablation surface is defined by zero of the hydrodynamic velocity. It separates the imploding inner part of the target and the expanding ablated plasma. The calculated ablation pressures creating both first and second shocks are $\sim 30 \%$ smaller compared to the values expected from the scaling relations [1]:

$$
P_{a b l}=12(A / 2 Z)^{1 / 3}\left(I_{a b s} / \lambda\right)^{2 / 3} \mathrm{Mbar},
$$

where $A$ and $Z$ are the average mass number and charge of the target, respectively, $\lambda$ is the laser wavelength in microns and $I_{a b s}$ is the absorbed laser intensity in units of $10^{14} \mathrm{~W} / \mathrm{cm}^{2}$. The increase in ablation pressure at the end of laser pulse at $t \sim 1.8 \mathrm{~ns}$ is explained by the reflected shock propagating outwards from the merging point.

The shock-wave dynamic is different if hot electrons are included in the simulation. First, according to Fig. 6, the ablation pressure is increases slightly to 120 to $130 \mathrm{Mbar}$. This is caused by the contribution of the low-energy part of the hot-electron spectrum, which is stopped in the transport zone before reaching the ablation surface. In contrast, the shock pressure is rising much stronger, first, to $\sim 180 \mathrm{Mbar}$, while propagating through the ablator, and then to 250 Mbar after merging with the first shock. As shown in Fig. 5, the shock propagates much faster in that case and 
(a)

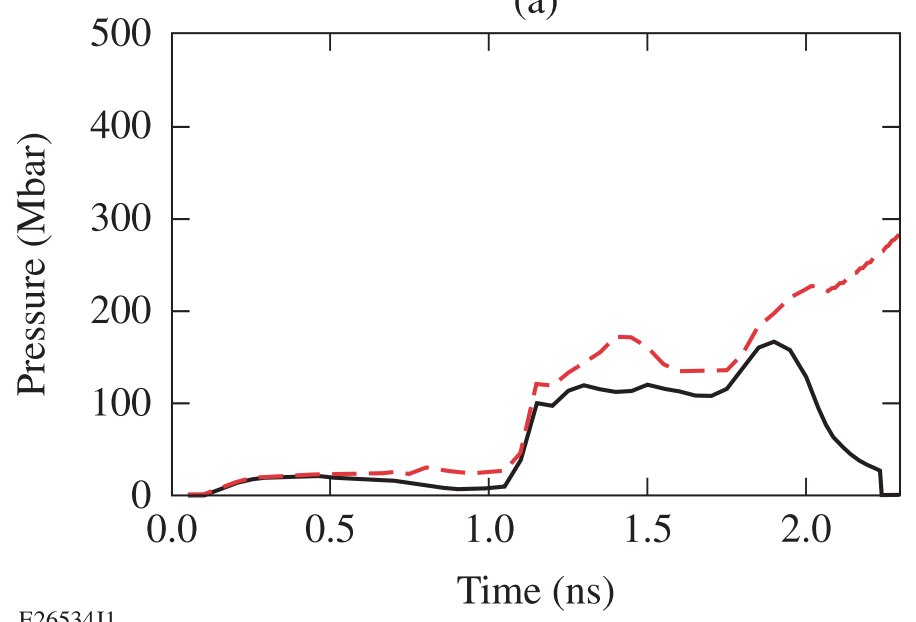

(b)

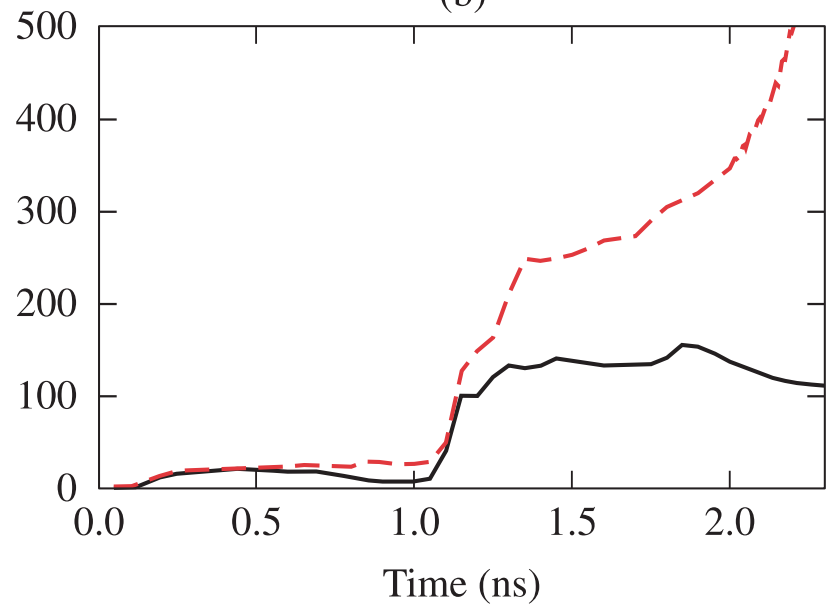

Figure 6. Evolution of the shock (red) and ablation (black) pressure for the case (a) without hot electrons and (b) with hot electrons.

its amplitude increases with time because of the convergence effect. It collapses at $t \simeq 2.4$ ns leading to the $\mathrm{x}$-ray flash shown in Fig. 4.

The boost in shock pressure from 150 to 250 Mbar resulting from the presence of hot electrons agrees with the qualitative estimate proposed in Refs. [6, 27]:

$$
P_{h e} \simeq 37 \rho_{t}^{1 / 3} I_{h e}^{2 / 3} \mathrm{Mbar},
$$

where $\rho_{t}$ is the target density downstream of the shock and $I_{h e}$ is the hot-electron energy flux in units of $10^{14} \mathrm{~W} / \mathrm{cm}^{2}$. For the target density behind the $4-\mathrm{g} / \mathrm{cm}^{3}$ shock and the electron energy flux of $3 \times 10^{14} \mathrm{~W} / \mathrm{cm}^{2}(8 \%$ of the incident laser intensity), one finds a pressure boost of $120 \mathrm{Mbar}$, which should be added to the shock pressure created by thermal electrons.

The situation is however more complicated: not all hot electrons deposit their energy downstream of the shock. An increase in the pressure can also be observed upstream of the shock. It is caused by the energy deposited by very energetic electrons that penetrate across the entire target. The areal density of the target is $40 \mathrm{mg} / \mathrm{cm}^{2}$, which corresponds to the stopping range of $200-\mathrm{keV}$ electrons. The SRS-generated electrons have an effective temperature of $40 \mathrm{keV}$; less than $1 \%$ of electrons have energies above this limit. Conversely, a significant part ( 6\%) of the TPD electrons with a temperature of $70 \mathrm{keV}$ may penetrate to the target center. However, the TPD electrons are strongly divergent and only a small fraction of them reach the central part of the target. To verify the origin of the target preheat, we conducted an additional simulation with the TPD switched off. This resulted in a less-than-5\% reduction in the shock pressure and less-than-50\% reduction in the temperature upstream of the shock, which prove the minor role of TPD in shock generation and target preheat. In agreement with the experiments $[11,12,21]$ the model confirms the dominant role of SRS-generated electrons in strong-shock dynamics.

Figure 7(a) shows the spatial and temporal evolution of the target temperature. It rises to 20 to $30 \mathrm{eV}$ at a distance of $\sim 50 \mu \mathrm{m}$ ahead the shock front. This results in a dramatic reduction of the shock strength, defined as a pressure ratio down- and upstream of the shock front, from more than 1000 in the case without hot electrons to about 10 in the case with hot electrons, as shown in Fig. 7(b). Knowing the specific heat capacity of $\mathrm{CH}$, which is $\sim 1 \mathrm{~J} / \mathrm{gK}$, and the target mass of $\sim 0.1 \mathrm{mg}$, we may estimate the energy deposited by hot electrons in the target upstream the shock. About $20 \mathrm{~J}$ energy is needed to heat the plastic to 20 to $30 \mathrm{eV}$, i.e., $1 \%$ of the total energy converted in the SRS-generated electrons. This number is indeed compatible with the hard electron fraction.

\section{CONCLUSION}

An analysis of a strong-shock experiment with numerical simulations taking into account the nonlinear laser-plasma interaction and hot electrons has provided a better understanding of the shock-wave dynamics driven by high-intensity laser pulses. First, a higher absorbed laser energy is correlated with a large fraction of hot electrons that are produced in the experiments with SSD off; $\sim 10 \%$ of the incident laser energy is transferred to hot electrons. SRS is the dominant nonlinear process producing hot electrons with a temperature of $40 \mathrm{keV}$. These electrons largely contribute 
(a)

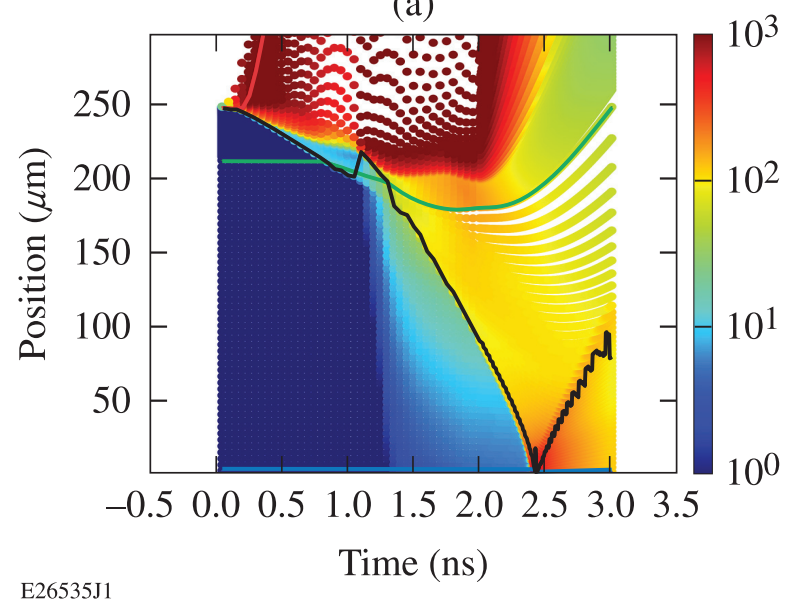

(b)

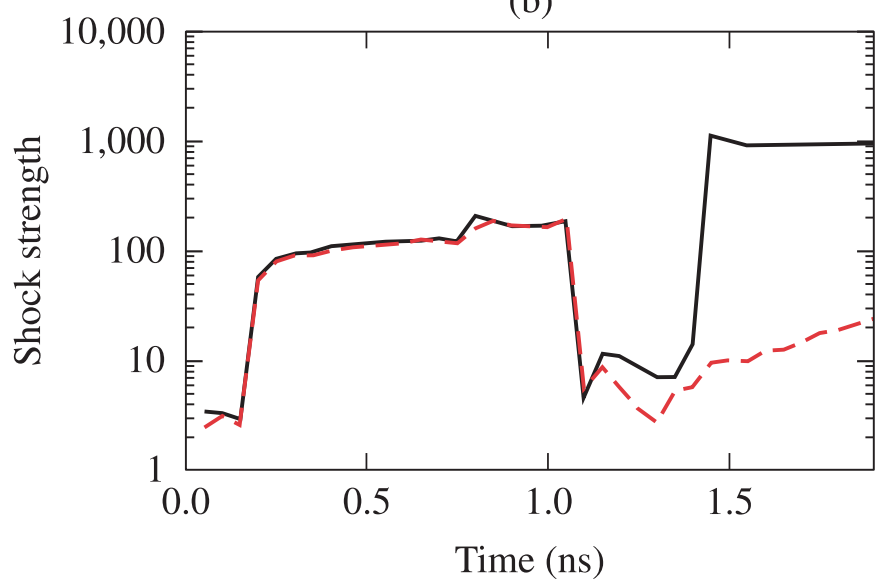

Figure 7. (a) Spatiotemporal evolution of the target temperature in the simulation with hot electrons. (b) The shock strength evolution for the runs without (black) and with (red) hot electrons.

to an increase in shock pressure by a factor of $\sim 2$ by depositing their energy downstream of the shock front. However, about $1 \%$ of the hot-electron energy is deposited upstream of the shock, significantly reducing its strength. Accounting for hot electrons in the simulation is found to be key to recovering measured experimental data, thereby allowing a more-robust estimation of the shock parameters.

The methodology proposed in Ref. [11] of estimating the shock pressure by changing the flux limiter in the case with hot electrons leads to a significant overestimate of the shock pressure and ablation pressure by 100 Mbar (or $30 \%$ ). The $C H I C$ simulations show only a minor increase in ablation pressure (less than 10\%) and a strong increase in shock pressure resulting from hot electrons. The target preheat is an undesirable issue in the context of shock ignition since a shock of a small strength is much less efficient in the fuel heating of the central spot and hot-electron preheat during the ignitor spike may lead to hot-spot mass increase because of inner-shell ablation [28]. These problems may be mitigated by designing a target with a larger areal density. Assuming the hot-electron temperature will be the same in the present shock-ignition target design, it would be sufficient to increase the areal shell density to 50 to $60 \mathrm{mg} / \mathrm{cm}^{2}$ at the moment of the shock launch to reduce the preheat by a factor of 10 and to restore the shock strength needed for ignition. Given the strong effects of hot electrons on shock dynamics, proposing a convincing shock-ignition design requires additional theoretical and experimental work. The most obvious problems are (1) obtaining a finer characterization of the hot-electron spectra generated by parametric instabilities, especially for the very high energy hot electrons, and (2) continuing the efforts to develop in-line models for coupling radiation-hydrodynamics codes with the generation of hot electrons. Additional work would also need to address the potential interplay between cross-beam energy transfer and the generation of hot electrons in direct-drive geometry.

\section{Acknowledgment}

This work has been carried out within the framework of the EUROfusion Consortium and has received funding from the Euratom research and training programme 2014-2018 under grant agreement No 633053. The views and opinions expressed herein do not necessarily reflect those of the European Commission.

We acknowledge a support from the Aquitaine Regional Council. Part of this work was supported by the DOE NNSA under awards No. DE-NA0001944 and DE-FC02-04ER54789, the Laboratory Basic Science Program, the University of Rochester, and the New York State Energy Research and Development Authority.

This report was prepared as an account of work sponsored by an agency of the U.S. Government. Neither the U.S. Government nor any agency thereof, nor any of their employees, makes any warranty, express or implied, or assumes any legal liability or responsibility for the accuracy, completeness, or usefulness of any information, apparatus, product, or process disclosed, or represents that its use would not infringe privately owned rights. Reference herein to any specific commercial product, process, or service by trade name, trademark, manufacturer, or otherwise does not necessarily constitute or imply its endorsement, recommendation, or favoring by the U.S. Government or any agency thereof. The views and opinions of authors expressed herein do not necessarily state or reflect those of the U.S. 
Government or any agency thereof.

[1] S. Atzeni, J. Meyer-ter-Vehn, "The Physics of Inertial Fusion," Oxford: Clarendon (2004).

[2] L. Spitzer, R. Härm, "Transport Phenomena in a Completely Ionized Gas," Phys. Rev. 89, 977 (1953).

[3] S. Braginskii, "Transport processes in a plasma," Reviews of Plasmas Physics. Consultants Bureau, New York, ed. by M. Leontovitch (1965).

[4] R. C. Malone, R. L. McCrory, R. L. Morse, "Indications of strongly flux-limited electron thermal conduction in laser-target experiments," Phys. Rev. Lett. 34, 721 (1975).

[5] G. P. Schurtz, Ph. D. Nicolaï, M. Busquet, "A nonlocal electron conduction model for multidimensional radiation hydrodynamics codes," Phys. Plasmas, 7, 4238 (2000).

[6] S. Gus'kov, X. Ribeyre, M. Touati, J.-L. Feugeas, P. Nicolaï, V. Tikhonchuk, "Ablation pressure driven by an energetic electron beam in a dense plasma," Phys. Rev. Lett. 109, 255004 (2012).

[7] X. Ribeyre, S. Gus'kov, J.-L. Feugeas, Ph. Nicolaï, V. T. Tikhonchuk, "Dense plasma heating and Gbar shock formation by a high intensity flux of energetic electrons," Phys. Plasmas 20, 062705 (2013).

[8] R. Betti, C. D. Zhou, K. S. Anderson, L. J. Perkins, W. Theobald, A. A. Solodov, "Shock ignition of thermonuclear fuel with high areal density," Phys. Rev. Lett. 98, 155001 (2007).

[9] D. Batani, S. Baton, A. Casner, S. Depierreux, M. Hohenberger, O. Klimo, M. Koenig, C. Labaune, X. Ribeyre, C. Rousseaux, G. Schurtz, W. Theobald, V. T. Tikhonchuk, "Physics issues for shock ignition," Nucl. Fusion 54, 054009 (2014).

[10] T. R. Boehly, D. L. Brown, R. S. Craxton, R. L. Keck, J. P. Knauer, J. H. Kelly, T. J. Kessler, S. A. Kumpan, S. J. Loucks, S. A. Letzring, F. J. Marshall, R. L. McCrory, S. F. B. Morse, W. Seka, J. M. Soures, C. P. Verdon, "Initial performance results of the OMEGA laser system," Opt. Commun. 133, 495 (1997).

[11] R. Nora, W. Theobald, R. Betti, F. J. Marshall, D. T. Michel, W. Seka, B. Yaakobi, M. Lafon, C. Stoeckl, J. Delettrez, A. A. Solodov, A. Casner, C. Reverdin, X. Ribeyre, A. Vallet, J. Peebles, F. N. Beg, M. S. Wei, "Gigabar spherical shock generation on the OMEGA laser," Phys. Rev. Lett. 114, 045001 (2015).

[12] W. Theobald, R. Nora, W. Seka, M. Lafon, K. S. Anderson, M. Hohenberger, F. J. Marshall, D. T. Michel, A. A. Solodov, C. Stoeckl, D. H. Edgell, B. Yaakobi, A. Casner, C. Reverdin, X. Ribeyre, A. Shvydky, A. Vallet, J. Peebles, F. N. Beg, M. S. Wei, R. Betti, "Spherical strong-shock generation for shock-ignition inertial fusion," Phys. Plasmas 22, 056310 (2015).

[13] J. Delettrez and E. B. Goldman, Laboratory for Laser Energetics, University of Rochester, Rochester, NY, LLE Report No. 36 (1976).

[14] A. A. Solodov and R. Betti, "Stopping power and range of energetic electrons in dense plasmas of fast-ignition fusion targets," Phys. Plasmas 15, 042707 (2008).

[15] J. Breil, S. Galera, P.-H. Maire, "Multi-material ALE computation in Inertial Confinement Fusion code CHIC," Comput. and Fluids 46,161 (2011).

[16] T. B. Kaiser, "Laser ray tracing and power deposition on an unstructured three-dimensional grid," Phys. Rev. E 61, 895 (2000).

[17] A. Colaïtis, G. Duchateau, P. Nicolaï, V. Tikhonchuk, "Towards modeling of nonlinear laser-plasma interactions with hydrocodes: the thick rays approach," Phys. Rev. E 89, 033101 (2014).

[18] A. Colaïtis, "Multiscale description of the laser-plasma interaction: application to the physics of shock ignition in inertial confinement fusion," Ph.D. thesis (University of Bordeaux, 2015).

[19] A. Colaïtis G. Duchateau, X. Ribeyre, Y. Maheut, G. Boutoux, L. Antonelli, Ph. Nicolaï, D. Batani, V. Tikhonchuk, "Coupled hydrodynamic model for laser-plasma interaction and hot electron generation," Phys. Rev. E 92041101 (2015).

[20] G. Cristoforetti, A. Colaïtis, L. Antonelli, S. Atzeni, F. Baffigi, D. Batani, F. Barbato, G. Boutoux, R. Dudzak, P. Koester, E. Krousky, L. Labate, Ph. Nicolaï, O. Renner, M. Skoric, V. Tikhonchuk, L. A. Gizzi, "Experimental observation of parametric instabilities at laser intensities relevant for shock ignition," Europhys. Lett. 117, 35001 (2017).

[21] W. Theobald, A. Bose, R. Yan, R. Betti, M. Lafon, D. Mangino, A. Christopherson, C. Stoeckl, W. Seka, W. Shang, D. T. Michel, C. Ren, R. C. Nora, A. Casner, J. Peebles, F. N. Beg, X. Ribeyre, E. Llor Aisa, A. Colaïtis, V. Tikhonchuk, M. S. Wei, "Enhanced hot-electron production and strong-shock generation in hydrogen rich ablators for shock ignition," Phys. Plasmas submitted.

[22] Y. A. Kravtsov, N. Y. Zhu, "Theory of Diffraction, Heuristic Approaches," Alpha Science Series on Wave Phenomena, Oxford (2010).

[23] C. Rousseaux, F. Amiranoff, C. Labaune, B. Mabille, G. Matthieussent, Rev. Phys. Appl. (Paris) 23, 1515 (1988).

[24] B. Yaakobi, A. A. Solodov, J. F. Myatt, J. A. Delettrez, C. Stoeckl, D. H. Froula, "Measurements of the divergence of fast electrons in laser-irradiated spherical targets," Phys. Plasmas 20, 092706 (2013).

[25] C. K. Li, R. D. Petrasso, "Stopping, straggling, and blooming of directed energetic electrons in hydrogenic and arbitrary- $Z$ plasmas," Phys. Rev. E 73, 016402 (2006).

[26] O. Peyrusse, "A model for the simulation of non-equilibrium line transfer in laboratory plasmas," Phys. Fluids B 4, 2007 (1992).

[27] E. Llor Aisa, X. Ribeyre, S. Yu. Gus'kov, V. T. Tikhonchuk, "The preplasma effect on the properties of the shock wave driven by a fast electron beam," Phys. Plasmas 23, 082702 (2016). 
[28] A. Colaïtis, X. Ribeyre, E. Le Bel, G. Duchateau, Ph. Nicolaï, V. Tikhonchuk, "Influence of laser induced hot electrons on the threshold for shock ignition of fusion reactions," Phys. Plasmas 23, 072703 (2016). 